\title{
Integrating brain methylome with GWAS for psychiatric risk gene discovery
}

Shizhong Han ${ }^{1}$, Ying Lin $^{2}$, Minghui Wang ${ }^{3}$, Fernando S. Goes ${ }^{1}$, Kai Tan ${ }^{4,5,6,7,8}$, Peter Zandi ${ }^{9}$, Thomas Hyde $^{10}$, Daniel R. Weinberger ${ }^{1,10,12,13}$, James B. Potash ${ }^{1}$, Joel E. Kleinman ${ }^{10}$, Andrew E. Jaffe ${ }^{9,10,11}$

${ }^{1}$ Department of Psychiatry and Behavioral Sciences, Johns Hopkins School of Medicine, Baltimore, MD, 21287, USA

${ }^{2}$ Department of Industrial Engineering, University of Houston, Houston, TX, 77204, USA

${ }^{3}$ Department of Genetics and Genomic Sciences, Icahn Institute of Genomics and Multiscale Biology, Icahn School of Medicine at Mount Sinai, New York, NY, 10029, USA

${ }^{4}$ Department of Genetics, Perelman School of Medicine, University of Pennsylvania, Philadelphia, PA, 19104, USA.

${ }^{5}$ Division of Oncology and Center for Childhood Cancer Research, Children's Hospital of Philadelphia, Philadelphia, PA, 19104, USA

${ }^{6}$ Department of Biomedical and Health Informatics, Children's Hospital of Philadelphia, Philadelphia, PA, 19104, USA

${ }^{7}$ Department of Pediatrics, Perelman School of Medicine, University of Pennsylvania, Philadelphia, PA, 19104, USA

${ }^{8}$ Department of Cell \& Developmental Biology, Perelman School of Medicine, University of Pennsylvania, Philadelphia, PA, 19104, USA

${ }^{9}$ Department of Mental Health, Johns Hopkins Bloomberg School of Public Health, Baltimore, MD, 21287, USA

${ }^{10}$ Lieber Institute for Brain Development, Johns Hopkins Medical Campus, Baltimore MD, 21287, USA

${ }^{11}$ Department of Biostatistics, Johns Hopkins Bloomberg School of Public Health, Baltimore, MD, 21287, USA

${ }^{12}$ Department of Neurology, Johns Hopkins School of Medicine, Baltimore, MD, 21287, USA 
${ }^{13}$ Departments of Neuroscience and the Institute of Genetic Medicine, Johns Hopkins School of Medicine, Baltimore, MD, 21287, USA

\section{Corresponding Author:}

Shizhong Han, $\mathrm{PhD}$,

Department of Psychiatry and Behavioral Sciences,

Johns Hopkins School of Medicine,

550 N. Broadway, Baltimore, Maryland, 21287, USA,

Tel: + 410-502-2733

Email: shan67@jhmi.edu 


\begin{abstract}
DNA methylation (DNAm) is heritable and plays a role in brain development and function through transcriptional regulation. Aberrant DNAm in human brain has been linked to psychiatric disorders, potentially as mediators of common genetic risk variants. In this study, we hypothesize that common risk variants for psychiatric disorders may act through affecting DNAm level in human brain. We first aimed to investigate the heritability pattern of DNAm levels in the human prefrontal cortex. Secondly, through imputation-driven methylome-wide association study (MWAS), we aimed to identify CpG sites whose methylation levels are genetically associated and that show methylation-trait associations in the prefrontal cortex of patients with schizophrenia or bipolar disorder. Our heritability analysis showed that, of $\sim 370,000 \mathrm{CpG}$ sites measured with the Illumina HumanMethylation450 microarray, $17 \%$ were heritable $(\mathrm{p}<0.05)$, with a mean heritability of 0.22 . Heritable $\mathrm{CpG}$ sites were enriched in intergenic regions, $\mathrm{CpG}$ shore, and regulatory regions in prefrontal cortex. Our MWAS approach identified known and potentially novel risk genes harboring $\mathrm{CpG}$ sites of methylation-trait associations for schizophrenia or bipolar disorder, which were not detectable using three alternative strategies (blood-based methylome reference, transcriptome-wide association study, and two gene-based association tests). Gene set enrichment analysis for genes with methylation-trait association evidence revealed pathways clearly related to neuronal functions, but also highlighted additional biological mechanisms that may underlie psychiatric disorders, such as microRNA-related regulation. In conclusion, our results showed the power of integrating brain methylation data with GWAS for psychiatric risk gene discovery, with potential applications in brain-related disorders or traits.
\end{abstract}




\section{Introduction}

DNA methylation (DNAm) is heritable and plays a critical role in brain development and function through transcriptional regulation $(1,2)$. Family and twin studies have investigated the heritability of DNAm for sites across the genome in easily accessible tissues (3-7), but, to our knowledge, there have been limited studies of methylation heritability in brain tissue. Indeed, only one study estimated the heritability of DNAm levels of individual CpG dinucleotides attributable to local single nucleotide polymorphisms (SNPs) in postmortem brain from unrelated individuals, but the estimation was limited to $\sim 21,000 \mathrm{CpG}$ sites primarily within promoters (8). The heritability pattern is not known for DNAm sites within gene body or intergenic regions, which represent a large portion of DNAm variation and are potentially important in epigenetic regulation of gene expression (9-12).

Aberrant DNAm has been linked to psychiatric disorders in candidate gene analysis and epigenome-wide association studies (EWAS) of schizophrenia (SCZ) $(13,14)$, bipolar disorder (BD) (15), and major depressive disorder $(16,17)$. However, the majority of these studies have been of easily accessible peripheral tissue (blood), which may not correlate with methylation signals in the brain $(18,19)$. Additionally, due to the challenges of accessing brain tissues, studies that examined brain samples were often limited by small sample size. Finally, it is hard to establish a causal role between DNAm change and disease as it is unclear whether aberrant DNAm causes disease or vice versa.

Genome-wide association studies (GWAS) indicate that most common genetic risk variants underlying complex diseases are noncoding and enriched in regulatory genomic regions in cells or tissues related to disease (20), suggesting that risk variants may act through regulation of cell/tissue-specific molecular mechanisms, such as methylation or gene expression. For example, SCZ risk variants from GWAS were enriched at enhancers active in brain, but not in tissues unlikely to be relevant to SCZ (21). Furthermore, statistical genetic approaches like linkage disequilibrium (LD) score regression have shown enrichment of 
trait heritability in regions with functional annotations that are trait-related and cell-type-specific, including enrichment of heritability for $\mathrm{SCZ}$ and $\mathrm{BP}$ in functional regions specific to the central nervous system (22) and even specific subclasses of neurons (23).

In line with a heritable component for DNAm, studies of methylation quantitaive trait loci (meQTL) have identified specific SNPs affecting methylation levels of CpG sites in human cell lines $(24,25)$, peripheral tissues $(26,27)$, and the human brain $(13,28,29)$. SNPs associated with meQTL largely act in cis and tend more often to be located at distant regulatory regions than at promoters (30, 31). Jaffe et al have shown that a large fraction of GWAS risk loci for SCZ contains an meQTL detected in adult frontal cortex (13). Significant enrichment of SCZ risk variants has also been observed among meQTLs calculated in prenatal brain tissue (29). It is therefore likely that DNAm may mediate the effect of genotype on disease risk for a proportion of risk variants.

In the current study, we first aimed to investigate the extent to which DNAm levels are determined by cisgenetic variations in human prefrontal cortex for $\sim 370,000 \mathrm{CpG}$ sites measured with the Illumina HumanMethylation450 microarray. Secondly, through imputation of methylation-trait association statistics from GWAS summary statistics, we aimed to identify CpG sites whose methylation is genetically associated, and that show methylation-trait associations in the prefrontal cortex for two major psychiatric disorders - SCZ and BD.

\section{Methods}

\section{Datasets}

DNAm and GWAS SNP genotyping data were available from postmortem dorsolateral prefrontal cortex brain tissue samples from 526 individuals. Methylation was measured using the Illumina 
HumanMethylation450 (450k) microarray, which measures CpG methylation across $>485,000$ probes. The current study used DNAm data from 238 unrelated subjects of European ancestry (100 SCZ patients and 138 controls), defined by principal component analysis (PCA) of GWAS data collected from the same samples. DNAm data were processed and normalized using the Minfi Bioconductor package in R (32). GWAS data were imputed into 1000 Genomes Phase 3 variants using SHAPEIT2 (33) and IMPUTE2 (34). After filtering out SNPs with MAF $<5 \%$, HWE p-value $<0.05$, or missing rate $>10 \%$, there were 4,402,285 SNPs on autosomes retained for further analyses. Information on tissue processing, experimental and bioinformatics procedures related to the methylation data, and genotype data processing was described in prior reports $(1,13)$.

We downloaded GWAS summary statistics from the Psychiatric Genomic Consortium (PGC) website (https://www.med.unc.edu/pgc/) for SCZ and BD. We then applied an imputation-driven methylomewide association study (MWAS) to the PGC2 GWAS meta-analysis summary statistics. We retained autosomal SNPs with MAF $>0.05$ and imputed quality score $>0.8$. Coordinates of SNPs were aligned to hg19 for all datasets.

\section{SNP-heritability of DNAm}

We estimated SNP-heritability of DNAm for CpG sites using GCTA software (35), which calculates the genetic relationship matrix (GRM) between subjects and uses the restricted maximum likelihood to estimate variance components in a mixed model framework. All pairs of 238 subjects with both GWAS and methylation data had a genetic relatedness $(\hat{\pi})$ less than 0.025 and were included for heritability estimation. GRM was calculated using SNPs within $1 \mathrm{Mb}$ of each $\mathrm{CpG}$ site. A number of covariates were included in the mixed model, including age, sex, SCZ diagnosis, the top five principal components (PCs) from the GWAS data to account for ancestry variability, and the top ten PCs from the methylation data (beta values) to control for cellular heterogeneity and latent batch effects. To avoid technical noise arising 
from SNPs within a probe region, we limited heritability estimation to $370,538 \mathrm{CpG}$ sites whose probes did not overlap with any SNPs based on annotations in the minfi Bioconductor package in R (32).

\section{CpG annotations}

To investigate the distribution of heritable $\mathrm{CpG}$ sites $(\mathrm{p}<0.05)$ across genomic features, we annotated CpG sites within three different contexts: 1) gene-based annotations from ANNOVAR (36), including exonic, splicing, ncRNA, 5'UTR, 3'UTR, intron, upstream (1 kb upstream of transcription start site), downstream (1 kb upstream of transcription end site), and intergenic; 2 ) annotations by distance to $\mathrm{CpG}$ island, including island, shelf ( $4 \mathrm{~kb}$ from islands), shore ( $\sim \mathrm{kb}$ from islands), and sea (others) using annotation information from the Illumina Infinium HM450 manifest file; 3) functional annotations of 15core chromatin states, derived using hidden Markov models, in the dorsolateral prefrontal cortex (E073) sample from the Epigenome Roadmap project (37). We used simulation-based approach to estimate the enrichment statistics of heritable $\mathrm{CpG}$ sites in each annotation category. Specifically, we first generated a background distribution from random $\mathrm{CpG}$ sets, while matching for $\mathrm{CpG}$ density found in heritable $\mathrm{CpG}$ sites. The enrichment fold was estimated by the ratio of the observed number of heritable CpGs overlapping with a specific annotation category to the average number of that from random $\mathrm{CpG}$ sets. The p-value for enrichment (depletion) was then the proportion of random $\mathrm{CpG}$ sets that fall in the same or a greater (smaller) number of annotations in each annotation category, as compared to the number found within heritable $\mathrm{CpG}$ set.

\section{Estimation of the cis-genetic component of methylation}

For each heritable CpG site ( $\mathrm{p}<0.05$ ), we first corrected its methylation level (beta value) by regressing out the same set of covariates as included in the heritability analysis. We then built models to predict the residual methylation levels using SNPs within $1 \mathrm{Mb}$ of each $\mathrm{CpG}$. We investigated three modeling 
schemes: top SNP, polygenic score, and elastic net. In the top SNP model, the SNP was simply chosen as the one with the strongest association with methylation. In the polygenic score model, we included all SNPs that were associated with methylation level at a significance level of $\mathrm{p}<0.05$, and the weights for the polygenic score were the effect sizes (beta coefficients from regression) estimated from the training sample. In the elastic net model, SNPs were selected using a penalized regression procedure that combines LASSO and ridge regression. The parameter $\alpha$ was tuned from 0 to 1 with a step increase by 0.1 . The final $\alpha$ value was determined as the one with the smallest prediction error from tenfold cross validation. To determine the optimal modeling scheme, we compared the fivefold cross-validated prediction $R^{2}$ values (the square of the correlation between predicted and observed methylation) for the three modelling schemes.

\section{Imputation-driven methylome-wide association study (MWAS)}

We performed MWAS to identify CpGs sites with methylation-trait association evidence by imputing GWAS summary statistics into methylation-trait association statistics for two psychiatric disorders SCZ and BD. To impute as many $\mathrm{CpG}$ sites as possible, we reintroduced $\mathrm{CpG}$ sites whose probes overlapped SNPs. Imputation was done for 49,442 CpG sites that were heritable $(\mathrm{p}<0.05)$ and had a cross-validated prediction $R^{2}>0.01$ by elastic net. Following the same theoretical framework as a transcriptome-wide association study (TWAS) (38), MWAS uses methylation data to compute weights for SNP association statistics from GWAS. In brief, based on our existing brain samples with both methylome and GWAS data, we used elastic net to estimate the genetic effects on DNAm level for SNPs within $1 \mathrm{Mb}$ of each $\mathrm{CpG}$ site. The methylation-trait association statistic was then calculated as a weighted linear combination of SNP-trait association statistics from GWAS, with weights being equal to estimated effect sizes on DNAm level from elastic net. Formally, let $\mathrm{Z}$ be a vector of the $\mathrm{Z}$-statistic for association between the GWAS trait and SNPs within $1 \mathrm{Mb}$ of a $\mathrm{CpG}$ site. We define $\mathrm{W}$ as a vector of weights that are effect sizes of the same set of SNPs on methylation level derived by elastic net. The imputed Z-statistic of 
methylation-trait association is then $W Z /\left(W \sum W\right)^{1 / 2}$, where $\sum$ is the correlation matrix among SNPs that can be estimated from an ancestry-matched reference sample. Bonferroni correction was used to control for multiple testing of 49,442 CpGs with a significance threshold of $\mathrm{p}=1 \times 10^{-6}$ for each disorder. We made the SNP weights on DNAm for each CpG site publicly available at: https://figshare.com/s/052a0b729c3c7ad7b535.

\section{TWAS and gene-based association tests}

We examined whether significant genes discovered by MWAS could be similarly uncovered by three alternative strategies. The first strategy utilized the MWAS framework, but used SNP weights derived from a blood-based methylome reference from http://mcn.unibas.ch/files/EstiMeth Distribution.zip (39). The second strategy was TWAS with the weights derived from gene expression data using dorsolateral prefrontal cortex, downloaded from the TWAS website (http://gusevlab.org/projects/fusion/\#referencefunctional-data). The third strategy involved two gene-level association tests for common variants, GATES (40) and VEGAS-sum (41). We assigned a SNP to a gene if it was located within the gene, based on NCBI 37.3 gene annotation, or within $20 \mathrm{~kb}$ upstream or downstream of the gene, to capture cis regulatory variants. GATES uses an extended Simes procedure to derive a gene-based p-value and is powerful when there is only one or a few causal SNPs. VEGAS-sum computes a gene-level p-value using simulations from a multivariate normal distribution and is powerful when there are multiple independent causal SNPs. Pairwise SNP correlations within a gene were estimated based on the genotype data of unrelated CEU samples from the 1000 Genomes Project. The total number of tests were 86,431 (CpGs), 5,419 (genes), and 24,583 (genes) for MWAS-blood, TWAS, and two gene-based tests, respectively. The Bonferroni-corrected significance threshold for each strategy was set at $\mathrm{p}=5.8 \times 10^{-7}$ (MWAS-blood), $\mathrm{p}=$ $9 \times 10^{-6}(\mathrm{TWAS})$, and $\mathrm{p}=2 \times 10^{-6}$ (GATES and VEGAS-sum). 


\section{Gene set enrichment analysis}

To understand the biology of genes with $\mathrm{CpG}$ sites of methylation-trait associations, we conducted gene set enrichment analysis using the logistic regression-based method LRpath (42), which relates the odds of gene set membership with the gene-level association signals, while adjusting for potentially confounding factors. Specifically, for each gene set, we defined the dependent variable y as 1 for genes in the set, and 0 for all other genes. The independent variable $\mathrm{x}$ is $-\log (\mathrm{p})$, where $\mathrm{p}$ is the gene-based $\mathrm{p}$ value defined by the smallest p-value among all imputed methylation-traits associations for $\mathrm{CpG}$ sites within a gene.

Logistic regression is used to model the log-odds of a gene belonging to a specific gene set as a function of $\mathrm{x}$. We included the number of imputed $\mathrm{CpG}$ sites within a gene as a covariate to control for potential confounding effect due to gene size difference. Gene sets were from two resources: 1) gene sets that are potentially important in psychiatric disorders (72 gene sets) (43), and 2) canonical pathways, Gene Ontology, and microRNA targets from MSigDB (v5.2) (5,986 gene sets) (44). To facilitate interpretation of the results, we included gene sets that overlapped at least 20, but not more than 2,000 genes with our tested genes. False discovery rate (FDR) q-values were calculated to account for multiple testing using the R statistical package "qvalue” (www.r-project.org).

We used hierarchical clustering to group significant gene sets into clusters based on similarity of their gene profiles (45). We first defined a gene overlapping matrix by counting the number of overlapping genes for each pair of gene sets. The Pearson correlation coefficient $R$ was then calculated for each pair of gene sets based on their overlap profiles. The distance matrix for hierarchical clustering was then $1-R$. Hierarchical clustering was performed using the "ward" method implemented in R function "hclust". The dendrogram and heatmap were plotted using the R function "heatmap.2". Clusters of related gene sets were manually determined to represent biologically relevant groups.

\section{Results}




\section{SNP-heritability of DNAm}

We estimated the SNP-heritability of DNAm levels for each CpG site using SNPs within $1 \mathrm{Mb}$. Figure 1a shows the distribution of heritability estimates for all, heritable $(\mathrm{p} \leq 0.05)$, and non-heritable $(\mathrm{p}>0.05)$ CpG sites separately. The average heritability was 0.050 across all $\mathrm{CpG}$ sites, heritable and nonheritable, ranging from 0 to 0.99 . There were 62,387 (17\%) CpG sites reaching a nominal level of significance for the heritability estimate $(\mathrm{p}<0.05)$, with an average heritability of 0.22 , ranging from 0.022 to 0.99 . Heritable CpG sites tended to be more continuous (Figure 1b) and had higher variance on average in methylation levels (beta-value) than non-heritable $\mathrm{CpG}$ sites (Wilcoxon rank sum test, $\mathrm{p}<2.2 \times 10^{-16}$ ) (Figure 1c). We investigated the distribution of heritable $\mathrm{CpG}$ sites across genomic features within different contexts. Heritable $\mathrm{CpG}$ sites were enriched in intergenic regions, $\mathrm{CpG}$ shore, and regulatory genomic regions (flanking active TSS and enhancers) in prefrontal cortex, but were depleted in 5'UTR, CpG islands, active TSS and strong transcription states in prefrontal cortex (Figure 1d).

We further estimated the SNP-heritability of DNAm levels using 100 neurotypical control samples. The heritability estimates were highly correlated between all samples and the neurotypical only samples $\left(R^{2}=\right.$ 0.85, Supplementary Figure 1). The average heritability in the neurotypical only sample was 0.062 across all CpG sites, which was slightly higher than the average estimates from the overall samples (0.05). Compared to the overall samples, there were fewer CpGs $(48,359,13 \%)$ reaching nominal significance for the heritability estimates $(\mathrm{p}<0.05)$, possibly due to the smaller sample size. In accordance with the pattern observed in the overall samples, heritable $\mathrm{CpG}$ sites tended to be more continuous and variable in methylation levels than non-heritable $\mathrm{CpG}$ sites, enriched in intergenic regions, $\mathrm{CpG}$ shore, flanking active TSS states in prefrontal cortex; heritable CpG sites were depleted in 5'UTR, CpG islands, and active TSS states in prefrontal cortex (Supplementary Figure 2). 


\section{Predicting the cis-genetic component of DNAm}

We evaluated whether the DNAm levels of heritable CpGs could be imputed from the genotype data for SNPs within $1 \mathrm{Mb}$ of the $\mathrm{CpG}$. We used fivefold cross-validation to compare predictive performance of three modeling schemes: top SNP, polygenic score and elastic net. Of the 32,949 CpGs for which all three models were successfully built, we found that the elastic net approach achieved the best performance with an average $R^{2}=0.24$, followed by the polygenic score $\left(R^{2}=0.16\right)$, and the top SNP methods $\left(R^{2}=0.095\right)$ (Figure 2a). When models were compared on the same CpG sites, $80 \%$ of the $\mathrm{CpG}$ sites achieved the best prediction by elastic net, whereas $18 \%$ and $2 \%$ of $\mathrm{CpG}$ sites were best predicted by the polygenic score and the top SNP methods, respectively (Figure 2b). Using heritability estimates as a benchmark for prediction $R^{2}$, we compared the prediction $R^{2}$ with the heritability estimate for each CpG (Figure 2c). We found that $89 \%$ of the CpGs achieved an $R^{2}$ equal to or larger than the lower bound of heritability estimate by elastic net, whereas 68\% (polygenic score) and 39\% (top SNP) of CpGs achieved such performance with the other methods . Overall, the elastic net approach showed the best performance for predicting DNAm levels based on different evaluation strategies. The effect sizes derived from elastic net were then used to impute differential methylation statistics from GWAS summary statistics.

\section{Imputation-driven methylome-wide association study (MWAS)}

We performed imputation-driven MWAS to identify CpGs sites of methylation-trait associations in prefrontal cortex for SCZ and BD. We imputed methylation-trait association statistics from PGC2 GWAS summary statistics for both disorders.

We identified 914 genome-wide significant (GWS) CpGs with differential methylation evidence for SCZ (Figure 3a, Supplementary Table 1). Of these, 883 overlapped original GWAS risk loci (defined by $1 \mathrm{Mb}$ upstream and downstream of any GWS SNPs from PGC2 SCZ GWAS); 677 CpGs were within the 
extended major histocompatibility complex region (chr6:25652464-33771788); 31 CpG sites were located more than $1 \mathrm{Mb}$ away from any GWS SNPs from PGC2 SCZ GWAS, suggesting potential novel signals not detected by GWAS. Table 1 shows details and methylation-trait association statistics for the $20 \mathrm{CpG}$ sites mapped to 24 genes. Of note, the gene MORC2-AS1 was far from GWS in PGC2 SCZ GWAS (smallest $\mathrm{p}=1.5 \times 10^{-5}, 1 \mathrm{Mb}$ window), but contained a $\mathrm{CpG}$ site at a gene body with strong evidence for methylation-trait association $\left(\operatorname{cg} 13896476, \mathrm{p}=3.4 \times 10^{-10}\right)$. Another $\mathrm{CpG}$ site, at promoter of MORC2-AS1, also showed significant evidence for methylation-trait association $(\operatorname{cg} 08837037, \mathrm{p}=$ $1.5 \times 10^{-8}$, Supplementary Table 1 ). Figure 4 shows the regional association plot of methylation-trait associations around MORC2-AS1, along with SNP association signals from GWAS and how their weights contribute to the top significant $\mathrm{CpG}$.

We identified 28 GWS CpGs with methylation-trait association evidence for BD (Figure 3b, Supplementary Table 2). Of these, 14 overlapped original GWAS risk loci and 14 were at least $1 \mathrm{Mb}$ away from any GWS SNPs. Table 2 shows details and methylation-trait association statistics for the 10 CpG sites mapped to 11 unique genes. Notably, $\mathrm{CNIH} 2$, which encodes a subunit of the ionotropic glutamate receptor in the human brain, showed strong evidence for methylation-trait association at a $\mathrm{CpG}$ site in the promoter region $\left(\operatorname{cg} 19026260, p=8.9 \times 10^{-9}\right)$, whereas the strongest GWAS signals within $1 \mathrm{Mb}$ of $C N I H 2$ was only at $\mathrm{p}=2.1 \times 10^{-7}$. Figure 5 shows the regional association plot of methylation-trait associations around $\mathrm{CNIH} 2$, along with SNP association signals from GWAS and how their weights contribute to the top significant $\mathrm{CpG}$.

\section{Comparison with alternative analytical strategies}

We evaluated three alternative strategies in their ability to detect the potential novel risk genes discovered above, which were at least $1 \mathrm{Mb}$ away from any GWS SNPs (Supplementary Table 3). Among the 24 potential novel risk genes from PGC2 SCZ, only five genes were detected: TMX2 (GATES, p = 6.9×10 ${ }^{-7}$; 
VEGAS-sum, $\left.\mathrm{p}=1.0 \times 10^{-6}\right)$, C11 orf 31 (VEGAS-sum, $\left.\mathrm{p}=1.0 \times 10^{-6}\right)$, CORO7 (TWAS, $\mathrm{p}=2.3 \times 10^{-7}$ ), TMTC1 (TWAS, $\mathrm{p}=1.8 \times 10^{-8}$ ), and BCL2L12 (VEGAS-sum, $\mathrm{p}=2.0 \times 10^{-6}$ ). For the ten potential novel risk genes from PGC2 BD, only PACS1 was identified (VEGAS-sum, $\mathrm{p}=1.0 \times 10^{-6}$ ).

\section{Gene set enrichment analysis}

We conducted gene set enrichment analyses to examine whether genes with methylation-trait associations clustered into biological functional groups. Full gene set analysis results are shown in Supplementary table 4 (SCZ) and Supplementary table 5 (BD).

Figure 6 shows summary results for 60 gene sets with enriched methylation-trait association evidence for SCZ (q-values $\leq 0.05)$. We grouped gene sets into eight major clusters. The highlighted gene sets in each cluster include: 1) genes involved in synaptic transmission and plasticity (dark red); 2) RBFOX1, RBFOX2, RBFOX3 regulatory networks, FMRP targets, synaptic genes, voltage-gated calcium channel transporters, missense constrained genes, mutation intolerant genes; SCZ candidate genes (green); 3) genes encoding voltage-gated calcium channels, genes related to membrane depolarization during action potential, genes associated with SCZ $\left(\mathrm{p}<10^{-4}\right)$, autism de novo genes (orange); 4) microRNA targets, SCZ de novo genes (blue); 5) microRNA targets, chromatin regulator CHD8 targets (red); 6) genes involved in antigen processing and presentation, genes associated with BP $\left(\mathrm{p}<10^{-4}\right)$ (brown); 7) genes involved in cell adhesion (black); 8) genes involved in axoneme assembly (dark blue).

Supplementary Figure 3 shows summary results for 51 gene sets with enriched methylation-trait association evidence for BD (q-values $\leq 0.05)$. Gene sets were grouped into five major clusters. The highlighted gene sets in each cluster include: 1) microRNA targets, SCZ de novo genes, and genes involved in cell adhesion (blue); 2) RBFOX1, RBFOX2, RBFOX3 regulatory networks, FMRP targets, synaptome, mutation intolerant genes (red); 3) genes involved in synaptic plasticity and transmission 
(green); 4) ion channel genes, missense constrained genes, calcium signaling pathway (purple); 5) genes involved in integrin signaling, genes associated with BP and depression $\left(\mathrm{p}<10^{-4}\right)$ (orange).

\section{Discussion}

In the current study, we have shown that DNAm levels are heritable for $\sim 17 \%$ of the CpG sites examined in human prefrontal cortex. Heritable DNAm sites tended to be more variable and enriched in intergenic and regulatory regions in brain. Through imputation of methylation-trait association from GWAS summary statistics, we identified known and potentially novel risk genes with methylation-trait association evidence that were not detectable using three alternative strategies. Gene set enrichment analysis for genes with methylation-trait association evidence revealed pathways related to neuronal functions, as well as other biological mechanisms potentially underlying psychiatric disorders, consistent with prior analyses based on GWAS (46) and gene expression data (47-49).

Heritability estimates of DNAm levels may vary across tissues and study designs. Twin studies reported an average heritability of $12-18 \%$ in whole blood $(7,50), 5 \%$ in placenta (7), and $19 \%$ in adipose tissue (28). A family study showed an average heritability of $13 \%$ in $\mathrm{CD} 4^{+}$cells (3). One study reported an average SNP-heritability of $29 \%$ across heritable CpG sites in colorectum (51). There has been to date only one study that estimated the SNP-heritability of DNAm in brain, but that was limited to 27,000 CpGs located primarily in promoters $(8)$. We found modest correlation of heritability estimates $(\mathrm{r}=0.48)$ for a common subset of CpGs between our study and the frontal cortex results of Quon et al (8). The variation of heritability estimates may be attributable to differences in brain samples and analytical approach. For example, we used a more narrowly defined single prefrontal cortical region---dorsolateral prefrontal cortex---from samples with a wide range of ages, whereas Quon et al used the whole frontal cortex largely from adults; we used SNPs within $1 \mathrm{Mb}$ window of CpGs, but Quon et al used a window size of $50 \mathrm{~kb}$. Importantly, our study differs from the previous one by virtue of a more than tenfold 
increase of CpG sites $(\sim 380,000)$ across a much broader portion of the genome, including intergenic and gene body regions. In addition, our study has a larger sample size than the previous one (238 versus 150$)$ and thus higher power to detect heritable $\mathrm{CpG}$ sites. We found a higher mean heritability for $\mathrm{CpG}$ sites in intergenic regions than for those at promoter regions. We also observed that heritable $\mathrm{CpG}$ sites tended to be located in intergenic regulatory genomic regions, suggesting a potentially important role in regulation of gene expression.

We evaluated three modeling schemes for their ability to predict the cis-genetic component of DNAm levels for $\mathrm{CpG}$ sites with a nominal significance level for heritability estimates. We found the highest performance for elastic net and the lowest performance for the top SNP method, suggesting that DNAm levels tend to be influenced by multiple SNPs. This observation is consistent with the result from a previous study in which elastic net achieved the best performance for predicting the cis-genetic component of gene expression using SNPs within $1 \mathrm{Mb}$ of a gene (52). Future work might employ other approaches to improve the prediction accuracy for DNAm levels. For example, recent work has included imputation of gene expression using the best linear predictor and the Bayesian linear mixed model (38), which can also be evaluated for DNAm prediction in the future.

Compared to a single marker association, the proposed MWAS features similar advantages as the TWAS method that aims to impute expression-trait association from GWAS summary statistics. First, both methods have significantly reduced multiple testing burdens compared to GWAS, though there are slightly more DNAm sites tested in MWAS than genes tested in TWAS. Second, because MWAS aims to detect methylation differences that are genetically driven, significant findings from MWAS may indicate a causal relationship between differential methylation and trait, whereas for EWAS the identified methylation difference may be a consequence of disease. This was further supported by the limited correlation $(\mathrm{r}=0.025)$ between the MWAS signals $(\mathrm{Z}$-score) from PGC SCZ2 GWAS and the EWAS signals (T-score) from the comparison of brains of SCZ versus controls identified in our previous study 
(13). Third, MWAS may increase the power to detect DNAm sites of small effect by taking advantage of GWAS summary statistics from large samples. In addition, MWAS has a unique advantage of higher coverage of genomic regions compared to TWAS. For example, MWAS covers CpGs in more than 10,000 genes in our study, whereas TWAS only contains $\sim 5,000$ genes for the prefrontal cortex region examined. Furthermore, DNAm levels may be a more proximal read out of genetic variation than gene expression levels, at least as measured in homogenate tissue at one time point (53), and MWAS may have greater power than TWAS in risk gene discovery in some situations.

We identified known and novel risk genes by applying MWAS to two major psychiatric disorders. We detected a number of potentially novel risk genes through application of MWAS to PGC2 SCZ GWAS (table 1). For example, KCNN3 was suspected to be involved in SCZ by candidate gene and gene expression studies (54-56); ANKS1B was among the top findings in a genome-wide pharmacogenomic study of antipsychotic treatment response in SCZ, which mediates the effect of quetiapine on working memory (57); TTBKl was found to harbor de novo mutations in sporadic cases of childhood onset SCZ (58); earlier linkage and candidate gene analysis showed the association evidence of BRD1 with SCZ (59), which was further supported by a recent functional study, demonstrating that $B R D 1$ regulates behavior, neurotransmission, and expression of SCZ risk-enriched gene sets in mice (60). The association between CNIH2 and BD is also noteworthy. CNIH2 encodes a subunit of the ionotropic glutamate receptor in the human brain, and has been shown to influence the efficacy of excitatory synaptic transmission (61).

Gene set enrichment analysis of MWAS association signals not only revealed pathways related to neuronal functions, such as synaptic genes and FMRP targets, but also highlighted other biological mechanisms that may underlie psychiatric disorders. One implication is the likely involvement of chromatin remodeling in SCZ as indicated by the enrichment of association signals in CHD8 targets. CHD8 is a DNA binding protein and acts as a chromatin remodeling factor. Genes targeted by CHD8 in human midfetal brain were enriched for functions related to transcriptional regulation and chromatin 
modification (62). CHD8 is also one of the most frequently mutated genes in autism (63-65), consistent with other data suggesting some genetic overlap between autism and SCZ (66). Perhaps the most notable highlight from gene set analysis is the involvement of microRNA-related regulation mechanism in psychiatric disorders. We found significant enrichment of microRNA targets for both disorders. Interestingly, targets of the hsa-miR-221 and hsa-miR-222 were found to be enriched for both disorders. Intriguingly, reduced expression of miR-221 was observed following chronic treatment with the antidepressant paroxetine in both rat hippocampus (67) and human lymphoblastoid cell lines (68). Additionally, we found significant enrichment of association signals in mutation-intolerant genes or missense constrained genes in both disorders. Our results are consistent with recent studies that have shown an enrichment of de novo mutations or rare risk variants in mutation-intolerant genes for neurodevelopmental disorders such as autism, intellectual disability and developmental delay, as well as SCZ (69-72).

This work should be viewed in light of several limitations. First, MWAS was designed to identify methylation-trait associations for DNAm sites that have a genetic component. It is therefore not suited to detect methylation differences caused exclusively by environmental factors. That being said, our heritability estimates themselves may be diluted by environmental factors. Indeed, the average heritability estimate from the overall samples was slightly lower than the estimate from the neurotypical samples alone, consistent with the possibility that systematic environmental factors (e.g. medical treatment) diminish heritability statistics in patients. Second, the number of DNAm sites that can be accurately imputed is limited by the training sample size and tissue types. Future studies will benefit from larger training samples and diverse tissues related to disease. Third, the Illumina CpG chip that we used is a relatively low resolution survey of the DNA methylation landscape, and though in includes many CpGs not in the 5' domain of genes and in $\mathrm{CpG}$ islands, the majority of $\mathrm{CpGs}$ are still in these regions. Fourth, MWAS can only identify risk SNPs that act through regulation of DNAm levels; it is not able to detect SNPs acting through mechanisms that are independent of DNAm regulation. Therefore, we view MWAS 
as by no means a replacement for current methods, such as TWAS or other gene-based tests, but as a complementary approach for uncovering genetic underpinnings underlying complex diseases.

In summary, we have shown the heritability pattern of DNAm in human prefrontal cortex. We further demonstrated the power of integrating the brain methylome with GWAS for psychiatric risk gene discovery, an approach which has potential applications in other brain-related disorders or traits.

\section{Acknowledgments}

The computations work for this study was supported by National Institutes of Health grants R01 AA022994 and AA024486 (to S.H.). All of the human brain data was generated from the Lieber Institute for Brain Development and supported by Institute funds. We are grateful for the vision and generosity of the Lieber and Maltz Families who made this work possible. We thank the families who donated to this research. We acknowledge the Psychiatric Genomics Consortium for making the GWAS results publicly available. 


\section{References}

1. COLANTUONI C, LIPSKA BK, YE T, et al. Temporal dynamics and genetic control of transcription in the human prefrontal cortex. Nature. 2011 Oct 26;478:519-23.

2. NUMATA S, YE T, HYDE TM, et al. DNA methylation signatures in development and aging of the human prefrontal cortex. American journal of human genetics. 2012 Feb 10;90:260-72.

3. DAY K, WAITE LL, ALONSO A, et al. Heritable DNA Methylation in CD4+ Cells among Complex Families Displays Genetic and Non-Genetic Effects. PloS one. 2016;11:e0165488.

4. VAN DONGEN J, NIVARD MG, WILLEMSEN G, et al. Genetic and environmental influences interact with age and sex in shaping the human methylome. Nature communications. $2016 \mathrm{Apr}$ 7;7:11115.

5. TREMBLAY BL, GUENARD F, LAMARCHE B, PERUSSE L, VOHL MC. Familial resemblances in blood leukocyte DNA methylation levels. Epigenetics. 2016 Nov;11:831-8.

6. GRUNDBERG E, MEDURI E, SANDLING JK, et al. Global analysis of DNA methylation variation in adipose tissue from twins reveals links to disease-associated variants in distal regulatory elements. American journal of human genetics. 2013 Nov 7;93:876-90.

7. GORDON L, JOO JE, POWELL JE, et al. Neonatal DNA methylation profile in human twins is specified by a complex interplay between intrauterine environmental and genetic factors, subject to tissue-specific influence. Genome research. 2012 Aug;22:1395-406.

8. QUON G, LIPPERT C, HECKERMAN D, LISTGARTEN J. Patterns of methylation heritability in a genome-wide analysis of four brain regions. Nucleic acids research. 2013 Feb 1;41:2095-104.

9. SCHUBELER D. Function and information content of DNA methylation. Nature. 2015 Jan 15;517:321-6.

10. BELL JT, PAI AA, PICKRELL JK, et al. DNA methylation patterns associate with genetic and gene expression variation in HapMap cell lines. Genome biology. 2011;12:R10.

11. GIBBS JR, VAN DER BRUG MP, HERNANDEZ DG, et al. Abundant quantitative trait loci exist for DNA methylation and gene expression in human brain. PLoS genetics. 2010 May 13;6:e1000952.

12. GUTIERREZ-ARCELUS M, LAPPALAINEN T, MONTGOMERY SB, et al. Passive and active DNA methylation and the interplay with genetic variation in gene regulation. eLife. 2013 Jun 4;2:e00523.

13. JAFFE AE, GAO Y, DEEP-SOBOSLAY A, et al. Mapping DNA methylation across development, genotype and schizophrenia in the human frontal cortex. Nature neuroscience. 2016 Jan;19:40-7.

14. MONTANO C, TAUB MA, JAFFE A, et al. Association of DNA Methylation Differences With Schizophrenia in an Epigenome-Wide Association Study. JAMA psychiatry. 2016 May 1;73:506-14. 15. LUDWIG B, DWIVEDI Y. Dissecting bipolar disorder complexity through epigenomic approach. Molecular psychiatry. 2016 Nov;21:1490-8.

16. SABUNCIYAN S, ARYEE MJ, IRIZARRY RA, et al. Genome-wide DNA methylation scan in major depressive disorder. PloS one. 2012;7:e34451.

17. JANUAR V, SAFFERY R, RYAN J. Epigenetics and depressive disorders: a review of current progress and future directions. International journal of epidemiology. 2015 Aug;44:1364-87.

18. SMITH AK, KILARU V, KLENGEL T, et al. DNA extracted from saliva for methylation studies of psychiatric traits: evidence tissue specificity and relatedness to brain. American journal of medical genetics Part B, Neuropsychiatric genetics : the official publication of the International Society of Psychiatric Genetics. 2015 Jan;168B:36-44.

19. HANNON E, LUNNON K, SCHALKWYK L, MILL J. Interindividual methylomic variation across blood, cortex, and cerebellum: implications for epigenetic studies of neurological and neuropsychiatric phenotypes. Epigenetics. 2015;10:1024-32. 
20. MAURANO MT, HUMBERT R, RYNES E, et al. Systematic localization of common diseaseassociated variation in regulatory DNA. Science. 2012 Sep 7;337:1190-5.

21. SCHIZOPHRENIA WORKING GROUP OF THE PSYCHIATRIC GENOMICS C. Biological insights from 108 schizophrenia-associated genetic loci. Nature. 2014 Jul 24;511:421-7.

22. FINUCANE HK, BULIK-SULLIVAN B, GUSEV A, et al. Partitioning heritability by functional annotation using genome-wide association summary statistics. Nature genetics. 2015 Nov;47:1228-35.

23. SKENE NG, BRYOIS J, BAKKEN TE, et al. Genetic identification of brain cell types underlying schizophrenia. Nature genetics. 2018 May 21.

24. BANOVICH NE, LAN X, MCVICKER G, et al. Methylation QTLS are associated with coordinated changes in transcription factor binding, histone modifications, and gene expression levels. PLoS genetics. 2014 Sep;10:e1004663.

25. ZHANG X, MOEN EL, LIU C, et al. Linking the genetic architecture of cytosine modifications with human complex traits. Human molecular genetics. 2014 Nov 15;23:5893-905.

26. GAUNT TR, SHIHAB HA, HEMANI G, et al. Systematic identification of genetic influences on methylation across the human life course. Genome biology. 2016 Mar 31;17:61.

27. MCCLAY JL, SHABALIN AA, DOZMOROV MG, et al. High density methylation QTL analysis in human blood via next-generation sequencing of the methylated genomic DNA fraction. Genome biology. 2015 Dec 23;16:291.

28. SCHULZ H, RUPPERT AK, HERMS S, et al. Genome-wide mapping of genetic determinants influencing DNA methylation and gene expression in human hippocampus. Nature communications. 2017 Nov 15;8:1511.

29. HANNON E, SPIERS H, VIANA J, et al. Methylation QTLs in the developing brain and their enrichment in schizophrenia risk loci. Nature neuroscience. 2016 Jan;19:48-54.

30. SHI J, MARCONETT CN, DUAN J, et al. Characterizing the genetic basis of methylome diversity in histologically normal human lung tissue. Nature communications. 2014 Feb 27;5:3365.

31. ZHANG D, CHENG L, BADNER JA, et al. Genetic control of individual differences in gene-specific methylation in human brain. American journal of human genetics. 2010 Mar 12;86:411-9.

32. ARYEE MJ, JAFFE AE, CORRADA-BRAVO $\mathrm{H}$, et al. Minfi: a flexible and comprehensive Bioconductor package for the analysis of Infinium DNA methylation microarrays. Bioinformatics. 2014 May 15;30:1363-9.

33. DELANEAU O, MARCHINI J, ZAGURY JF. A linear complexity phasing method for thousands of genomes. Nature methods. 2011 Dec 4;9:179-81.

34. HOWIE B, FUCHSBERGER C, STEPHENS M, MARCHINI J, ABECASIS GR. Fast and accurate genotype imputation in genome-wide association studies through pre-phasing. Nature genetics. $2012 \mathrm{Jul}$ 22;44:955-9.

35. YANG J, LEE SH, GODDARD ME, VISSCHER PM. GCTA: a tool for genome-wide complex trait analysis. American journal of human genetics. 2011 Jan 7;88:76-82.

36. WANG K, LI M, HAKONARSON H. ANNOVAR: functional annotation of genetic variants from highthroughput sequencing data. Nucleic acids research. 2010 Sep;38:e164.

37. ROADMAP EPIGENOMICS C, KUNDAJE A, MEULEMAN W, et al. Integrative analysis of 111 reference human epigenomes. Nature. 2015 Feb 19;518:317-30.

38. GUSEV A, KO A, SHI H, et al. Integrative approaches for large-scale transcriptome-wide association studies. Nature genetics. 2016 Mar;48:245-52.

39. FREYTAG V, VUKOJEVIC V, WAGNER-THELEN H, et al. Genetic estimators of DNA methylation provide insights into the molecular basis of polygenic traits. Translational psychiatry. 2018 Jan 31;8:31. 40. LI MX, GUI HS, KWAN JS, SHAM PC. GATES: a rapid and powerful gene-based association test using extended Simes procedure. American journal of human genetics. 2011 Mar 11;88:283-93. 
41. LIU JZ, MCRAE AF, NYHOLT DR, et al. A versatile gene-based test for genome-wide association studies. American journal of human genetics. 2010 Jul 9;87:139-45.

42. SARTOR MA, LEIKAUF GD, MEDVEDOVIC M. LRpath: a logistic regression approach for identifying enriched biological groups in gene expression data. Bioinformatics. 2009 Jan 15;25:211-7.

43. GASPAR HA, BREEN G. Drug enrichment and discovery from schizophrenia genome-wide association results: an analysis and visualisation approach. Scientific reports. 2017 Sep 29;7:12460.

44. SUBRAMANIAN A, TAMAYO P, MOOTHA VK, et al. Gene set enrichment analysis: a knowledgebased approach for interpreting genome-wide expression profiles. Proceedings of the National Academy of Sciences of the United States of America. 2005 Oct 25;102:15545-50.

45. CHEN YA, TRIPATHI LP, DESSAILLY BH, NYSTROM-PERSSON J, AHMAD S, MIZUGUCHI K. Integrated pathway clusters with coherent biological themes for target prioritisation. PloS one. 2014;9:e99030.

46. NETWORK, PATHWAY ANALYSIS SUBGROUP OF PSYCHIATRIC GENOMICS C. Psychiatric genomewide association study analyses implicate neuronal, immune and histone pathways. Nature neuroscience. $2015 \mathrm{Feb}$;8:199-209.

47. BARNES MR, HUXLEY-JONES J, MAYCOX PR, et al. Transcription and pathway analysis of the superior temporal cortex and anterior prefrontal cortex in schizophrenia. Journal of neuroscience research. 2011 Aug;89:1218-27.

48. SANTARELLI DM, BEVERIDGE NJ, TOONEY PA, CAIRNS MJ. Upregulation of dicer and microRNA expression in the dorsolateral prefrontal cortex Brodmann area 46 in schizophrenia. Biological psychiatry. 2011 Jan 15;69:180-7.

49. DARBY MM, YOLKEN RH, SABUNCIYAN S. Consistently altered expression of gene sets in postmortem brains of individuals with major psychiatric disorders. Translational psychiatry. 2016 Sep 13;6:e890.

50. BELL JT, TSAI PC, YANG TP, et al. Epigenome-wide scans identify differentially methylated regions for age and age-related phenotypes in a healthy ageing population. PLoS genetics.

2012;8:e1002629.

51. ROWLATT A, HERNANDEZ-SUAREZ G, SANABRIA-SALAS MC, et al. The heritability and patterns of DNA methylation in normal human colorectum. Human molecular genetics. 2016 Jun 15;25:2600-11.

52. GAMAZON ER, WHEELER HE, SHAH KP, et al. A gene-based association method for mapping traits using reference transcriptome data. Nature genetics. 2015 Sep;47:1091-8.

53. KLEINMAN JE, LAW AJ, LIPSKA BK, et al. Genetic neuropathology of schizophrenia: new approaches to an old question and new uses for postmortem human brains. Biological psychiatry. 2011 Jan 15;69:140-5.

54. CHANDY KG, FANTINO E, WITTEKINDT O, et al. Isolation of a novel potassium channel gene hSKCa3 containing a polymorphic CAG repeat: a candidate for schizophrenia and bipolar disorder? Molecular psychiatry. 1998 Jan;3:32-7.

55. DROR V, SHAMIR E, GHANSHANI S, et al. hKCa3/KCNN3 potassium channel gene: association of longer CAG repeats with schizophrenia in Israeli Ashkenazi Jews, expression in human tissues and localization to chromosome 1q21. Molecular psychiatry. 1999 May;4:254-60.

56. MARTIN S, LAZZARINI M, DULLIN C, et al. SK3 Channel Overexpression in Mice Causes Hippocampal Shrinkage Associated with Cognitive Impairments. Molecular neurobiology. 2017 Mar;54:1078-91.

57. MCCLAY JL, ADKINS DE, ABERG K, et al. Genome-wide pharmacogenomic study of neurocognition as an indicator of antipsychotic treatment response in schizophrenia.

Neuropsychopharmacology : official publication of the American College of Neuropsychopharmacology. 2011 Feb;36:616-26. 
58. AMBALAVANAN A, GIRARD SL, AHN K, et al. De novo variants in sporadic cases of childhood onset schizophrenia. European journal of human genetics : EJHG. 2016 Jun;24:944-8.

59. SEVERINSEN JE, BJARKAM CR, KIAER-LARSEN S, et al. Evidence implicating BRD1 with brain development and susceptibility to both schizophrenia and bipolar affective disorder. Molecular psychiatry. 2006 Dec;11:1126-38.

60. QVIST P, CHRISTENSEN JH, VARDYA I, et al. The Schizophrenia-Associated BRD1 Gene Regulates Behavior, Neurotransmission, and Expression of Schizophrenia Risk Enriched Gene Sets in Mice. Biological psychiatry. 2017 Jul 1;82:62-76.

61. BOUDKKAZI S, BRECHET A, SCHWENK J, FAKLER B. Cornichon2 dictates the time course of excitatory transmission at individual hippocampal synapses. Neuron. 2014 May 21;82:848-58.

62. COTNEY J, MUHLE RA, SANDERS SJ, et al. The autism-associated chromatin modifier CHD8 regulates other autism risk genes during human neurodevelopment. Nature communications. $2015 \mathrm{Mar}$ 10;6:6404.

63. O'ROAK BJ, VIVES L, FU W, et al. Multiplex targeted sequencing identifies recurrently mutated genes in autism spectrum disorders. Science. 2012 Dec 21;338:1619-22.

64. BERNIER R, GOLZIO C, XIONG B, et al. Disruptive CHD8 mutations define a subtype of autism early in development. Cell. 2014 Jul 17;158:263-76.

65. O'ROAK BJ, STESSMAN HA, BOYLE EA, et al. Recurrent de novo mutations implicate novel genes underlying simplex autism risk. Nature communications. 2014 Nov 24;5:5595.

66. CARROLL LS, OWEN MJ. Genetic overlap between autism, schizophrenia and bipolar disorder. Genome medicine. 2009 Oct 30;1:102.

67. ZHOU R, YUAN P, WANG Y, et al. Evidence for selective microRNAs and their effectors as common long-term targets for the actions of mood stabilizers. Neuropsychopharmacology : official publication of the American College of Neuropsychopharmacology. 2009 May;34:1395-405.

68. OVED K, MORAG A, PASMANIK-CHOR M, REHAVI M, SHOMRON N, GURWITZ D. Genome-wide expression profiling of human lymphoblastoid cell lines implicates integrin beta- 3 in the mode of action of antidepressants. Translational psychiatry. 2013 Oct 15;3:e313.

69. DECIPHERING DEVELOPMENTAL DISORDERS S. Prevalence and architecture of de novo mutations in developmental disorders. Nature. 2017 Feb 23;542:433-8.

70. KOSMICKI JA, SAMOCHA KE, HOWRIGAN DP, et al. Refining the role of de novo proteintruncating variants in neurodevelopmental disorders by using population reference samples. Nature genetics. 2017 Apr;49:504-10.

71. SAMOCHA KE, ROBINSON EB, SANDERS SJ, et al. A framework for the interpretation of de novo mutation in human disease. Nature genetics. 2014 Sep;46:944-50.

72. GENOVESE G, FROMER M, STAHL EA, et al. Increased burden of ultra-rare protein-altering variants among 4,877 individuals with schizophrenia. Nature neuroscience. 2016 Nov;19:1433-41. 
Figure 1. SNP-based heritability analysis of DNAm levels: a) Distribution of heritability estimates for all, heritable $(\mathrm{p} \leq 0.05)$, and non-heritable $(\mathrm{p}>0.05)$ CpG sites; $\mathrm{b})$ Distribution of methylation levels (betavalue) for all, heritable $(\mathrm{p} \leq 0.05)$, and non-heritable $(\mathrm{p}>0.05) \mathrm{CpG}$ sites; $\mathrm{c})$ Boxplot for variance of methylation levels (beta-value) for heritable $(\mathrm{p} \leq 0.05)$ and non-heritable $(\mathrm{p}>0.05) \mathrm{CpG}$ sites; $\mathrm{d})$ Enrichment of heritable $\mathrm{CpG}$ sites across genomic features within three different contexts: genomic locations (blue), distance to $\mathrm{CpG}$ islands (grey), and functional states in the dorsolateral prefrontal cortex (red). The x-axis represents enrichment fold. The y-axis labels the different features examined. The dotted yellow line indicates no enrichment (fold $=1$ ). The numbers next to each bar are enrichment p-values.

Figure 2. Evaluation of prediction performance $\left(R^{2}\right)$ for three modeling schemes: a) Boxplot of prediction performance $\left(R^{2}\right)$ for three modeling schemes. Red points represent the mean value of $R^{2}$ across CpG sites; b) Barplot for the proportion of CpGs achieving the best prediction performance under each modeling scheme; c) Comparison of prediction performance $\left(R^{2}\right)$ with heritability estimates $\left(h^{2}\right)$. The figure shows the cross-validated prediction performance ( $R^{2}$ in red) in comparison to methylation heritability estimates (black). The gray zone shows the $95 \%$ confidence intervals of heritability estimates.

Figure 3. Genome-wide association plot for methylation-trait associations for schizophrenia and bipolar disorder (PGC2). The dotted red line indicates significance thresholds. Red points represent significant CpG sites that are at least $1 \mathrm{Mb}$ away from genomewide significant SNPs.

Figure 4. Regional association plot around the top significant $\mathrm{CpG}$ within MORCI-AS. The upper panel shows gene names and locations based on hg19 coordinates. The middle panel shows association strength of top significant $\mathrm{CpG}$ (blue diamond) and surrounding $\mathrm{CpG}$ sites (black) within $1 \mathrm{Mb}$. The bottom panel shows association signals of SNPs from GWAS at the same region. SNPs are colored according to their weights contributing to the top significant $\mathrm{CpG}$. 
Figure 5. Regional association plot around the top significant $\mathrm{CpG}$ within $C N I H 2$. The upper panel shows gene names and locations based on hg19 coordinates. The middle panel shows association strength of top significant $\mathrm{CpG}$ (blue diamond) and surrounding $\mathrm{CpG}$ sites (black) within $1 \mathrm{Mb}$. The bottom panel shows association signals of SNPs from GWAS at the same region. SNPs are colored according to their weights contributing to the top significant $\mathrm{CpG}$.

Figure 6. Hierarchical clustering of significant gene sets in schizophrenia. Gene sets were grouped into eight clusters as indicated by different colors. The color gradient indicates the enrichment p-values of each gene set as shown in the middle panel. 
Table 1. Information and methylation-trait association statistics for CpGs associated with schizophrenia

\begin{tabular}{|c|c|c|c|c|c|c|c|}
\hline CpGs & Chr & Position & Gene & Function & $\mathbf{z}$ & $\mathbf{p}$ & p $_{\text {smallest }}{ }^{*}$ \\
\hline ch.1.1029351R & 1 & 31734918 & SNRNP4O & Body & -5.66 & $1.50 \mathrm{E}-08$ & $3.51 \mathrm{E}-06$ \\
\hline $\operatorname{cg} 06221963$ & 1 & 154839813 & KCNN3 & Body & -5.01 & $5.31 \mathrm{E}-07$ & $3.18 \mathrm{E}-06$ \\
\hline cg24077277 & 1 & 205201540 & $T M C C 2$ & Body & -4.95 & $7.28 \mathrm{E}-07$ & $8.69 \mathrm{E}-07$ \\
\hline $\operatorname{cg} 19875535$ & 5 & 140030758 & $I K$ & Body & -5.40 & $6.51 \mathrm{E}-08$ & $4.28 \mathrm{E}-07$ \\
\hline $\operatorname{cg} 06503255$ & 5 & 140053350 & DNDI & TSS200 & -4.97 & $6.77 \mathrm{E}-07$ & $4.28 \mathrm{E}-07$ \\
\hline cg25984996 & 5 & 140098398 & VTRNAl-2 & TSS200 & 5.18 & 2.19E-07 & $4.28 \mathrm{E}-07$ \\
\hline $\operatorname{cg} 07539045$ & 6 & 43245571 & TTBK1 & Body & -5.33 & $9.85 \mathrm{E}-08$ & $9.61 \mathrm{E}-07$ \\
\hline $\operatorname{cg} 13305186$ & 11 & 57508416 & $\begin{array}{l}\text { TMX2;TMX2; } \\
\text { C11 orf } 31\end{array}$ & $\begin{array}{l}\text { 3'UTR;Body; } \\
\text { TSS1500 }\end{array}$ & 4.95 & $7.56 \mathrm{E}-07$ & $6.65 \mathrm{E}-08$ \\
\hline $\operatorname{cg} 25744127$ & 11 & 109292505 & C11orf87 & TSS1500 & -4.99 & $6.12 \mathrm{E}-07$ & $1.72 \mathrm{E}-07$ \\
\hline cg14258853 & 12 & 29935411 & TMTCl & 5'UTR & 5.18 & $2.25 \mathrm{E}-07$ & $7.06 \mathrm{E}-08$ \\
\hline $\operatorname{cg} 24305861$ & 12 & 99564273 & $A N K S I B$ & Body & -4.92 & $8.61 \mathrm{E}-07$ & $1.28 \mathrm{E}-06$ \\
\hline $\operatorname{cg} 09069446$ & 16 & 4462122 & CORO7 & Body & 4.93 & $8.21 \mathrm{E}-07$ & $2.79 \mathrm{E}-07$ \\
\hline $\operatorname{cg} 08460995$ & 16 & 4526227 & $\begin{array}{l}\text { HMOX2;HMOX2; } \\
\text { NMRAL1 }\end{array}$ & $\begin{array}{l}\text { TSS200;5'UTR; } \\
\text { TSS1500 }\end{array}$ & -5.03 & $4.87 \mathrm{E}-07$ & $2.79 \mathrm{E}-07$ \\
\hline cg26619894 & 17 & 19249164 & MIRI180;B9DI & TSS1500;Body & 4.98 & $6.40 \mathrm{E}-07$ & 7.77E-07 \\
\hline cg22598241 & 19 & 2131519 & $A P 3 D 1$ & Body & -5.29 & $1.24 \mathrm{E}-07$ & $1.22 \mathrm{E}-06$ \\
\hline $\operatorname{cg} 04052466$ & 19 & 2251061 & $A M H$ & Body & 5.16 & $2.50 \mathrm{E}-07$ & $1.22 \mathrm{E}-06$ \\
\hline $\operatorname{cg} 09250473$ & 19 & 50168907 & $\begin{array}{l}\text { IRF3;IRF3; } \\
\text { BCL2LI2;BCL2L12 }\end{array}$ & $\begin{array}{l}\text { 1stExon;5'UTR; } \\
\text { 5'UTR;1stExon }\end{array}$ & -4.99 & $6.06 \mathrm{E}-07$ & $2.19 \mathrm{E}-07$ \\
\hline $\operatorname{cg} 13896476$ & 22 & 31318546 & MORC2-AS1 & Body & -6.28 & $3.37 \mathrm{E}-10$ & $1.46 \mathrm{E}-05$ \\
\hline cg22416021 & 22 & 50177941 & $B R D 1$ & Body & 5.25 & $1.53 \mathrm{E}-07$ & $3.31 \mathrm{E}-07$ \\
\hline $\operatorname{cg} 26441486$ & 22 & 50317300 & CRELD2 & Body & 5.17 & $2.36 \mathrm{E}-07$ & $3.31 \mathrm{E}-07$ \\
\hline
\end{tabular}

* the strongest GWAS signals from SNPs within $1 \mathrm{Mb}$ of each gene 
Table 2. Information and methylation-trait association statistics for CpGs associated with bipolar disorder

\begin{tabular}{|l|l|l|l|l|l|l|l|}
\hline CpGs & Chr & Position & Gene & Function & $\mathbf{z}$ & $\mathbf{p}$ & p $_{\text {smallest }}{ }^{*}$ \\
\hline $\operatorname{cg} 20822109$ & 3 & 183146412 & MCF2L2 & TSS1500 & 5.45 & $5.04 \mathrm{E}-08$ & $4.25 \mathrm{E}-05$ \\
\hline $\operatorname{cg} 17797898$ & 7 & 1950858 & MAD1L1 & Body & -4.93 & $8.36 \mathrm{E}-07$ & $2.38 \mathrm{E}-06$ \\
\hline $\operatorname{cg} 09700701$ & 11 & 65816819 & GAL3ST3 & TSS200 & 4.94 & $8.01 \mathrm{E}-07$ & $2.08 \mathrm{E}-07$ \\
\hline $\operatorname{cg} 14541915$ & 11 & 65894463 & PACS1 & Body & -5.01 & $5.51 \mathrm{E}-07$ & $2.08 \mathrm{E}-07$ \\
\hline $\operatorname{cg} 19026260$ & 11 & 66045351 & CNIH2 & TSS1500 & 5.75 & $8.88 \mathrm{E}-09$ & $2.08 \mathrm{E}-07$ \\
\hline $\operatorname{cg} 03423112$ & 11 & 66335975 & CTSF;CTSF & 5'UTR;1stExon & 5.10 & $3.42 \mathrm{E}-07$ & $2.08 \mathrm{E}-07$ \\
\hline $\operatorname{cg} 00757327$ & 12 & 49390906 & DDN & Body & 5.02 & $5.06 \mathrm{E}-07$ & $3.25 \mathrm{E}-07$ \\
\hline $\operatorname{cg} 03568305$ & 17 & 38183559 & MED24;SNORD124 & Body;TSS1500 & 5.82 & $6.05 \mathrm{E}-09$ & $4.50 \mathrm{E}-07$ \\
\hline $\operatorname{cg} 25909396$ & 17 & 64300729 & PRKCA & Body & -5.32 & $1.04 \mathrm{E}-07$ & $6.25 \mathrm{E}-06$ \\
\hline $\operatorname{cg} 06409856$ & 19 & 10750786 & SLC44A2 & Body & -5.00 & $5.62 \mathrm{E}-07$ & $1.60 \mathrm{E}-07$ \\
\hline
\end{tabular}

* the strongest GWAS signals from SNPs within $1 \mathrm{Mb}$ of each gene 
Supplementary Figure 1. Scatter plot for heritability estimates from the overall samples and neurotypical samples.

Supplementary Figure 2. SNP-based heritability analysis of DNAm levels from neurotypical samples: a) Distribution of heritability estimates for all, heritable $(p \leq 0.05)$, and non-heritable $(p>0.05)$ CpG sites; $b)$ Distribution of methylation levels (beta-value) for all, heritable $(\mathrm{p} \leq 0.05)$, and non-heritable $(\mathrm{p}>0.05)$ CpG sites; $c)$ Boxplot for variance of methylation levels (beta-value) for heritable $(\mathrm{p} \leq 0.05)$ and nonheritable ( $\mathrm{p}>0.05$ ) $\mathrm{CpG}$ sites; d) Enrichment of heritable $\mathrm{CpG}$ sites across genomic features within three different contexts: genomic locations (blue), distance to $\mathrm{CpG}$ islands (grey), and functional states in the dorsolateral prefrontal cortex (red). The x-axis represents enrichment fold. The y-axis labels the different features examined. The dotted yellow line indicates no enrichment (fold $=1$ ). The numbers next to each bar are enrichment p-values.

Supplementary Figure 3. Hierarchical clustering of significant gene sets in bipolar disorder. Gene sets were grouped into five clusters as indicated by different colors. The color gradient indicates the enrichment p-values of each gene set as shown in the middle panel. 

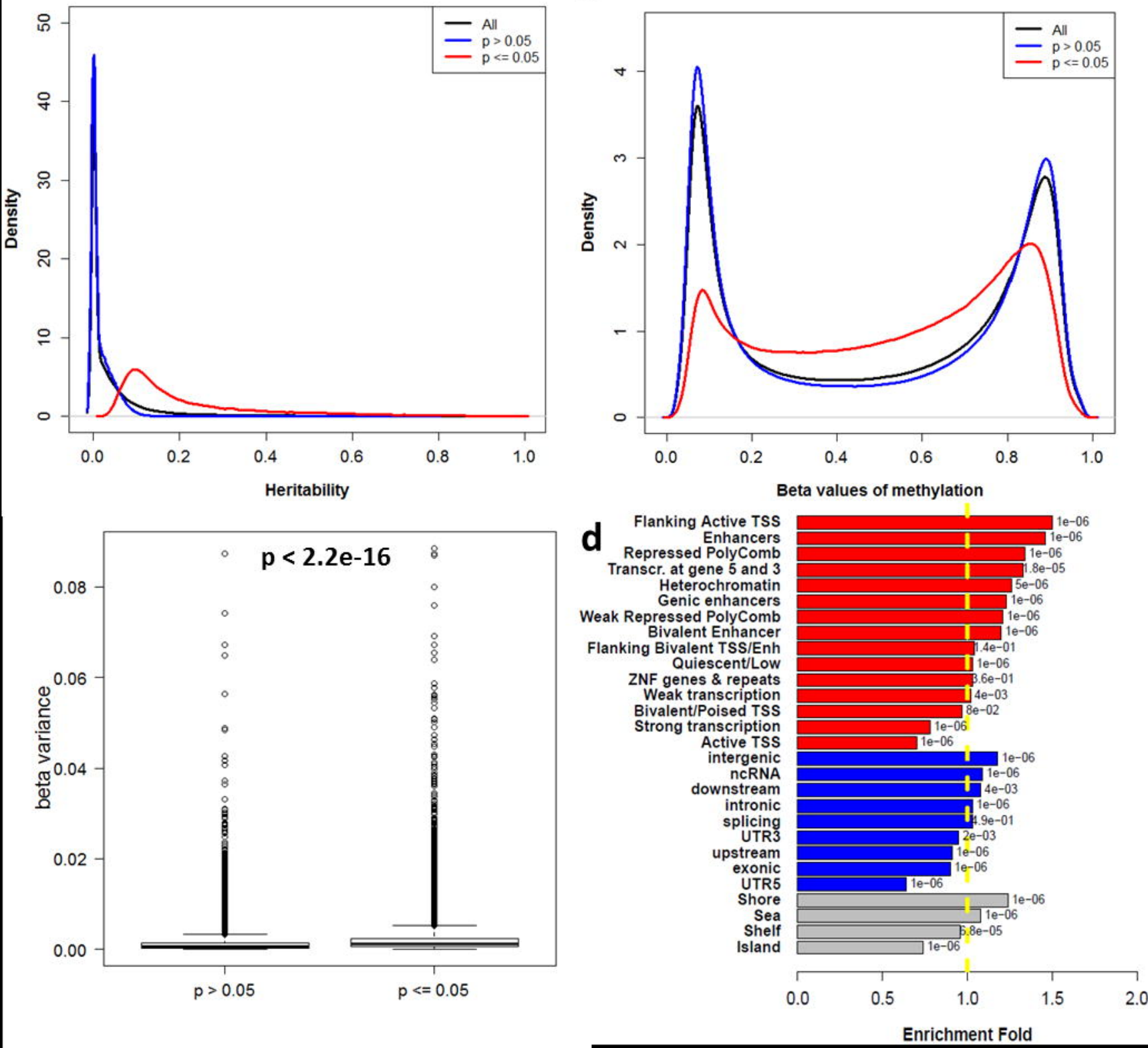


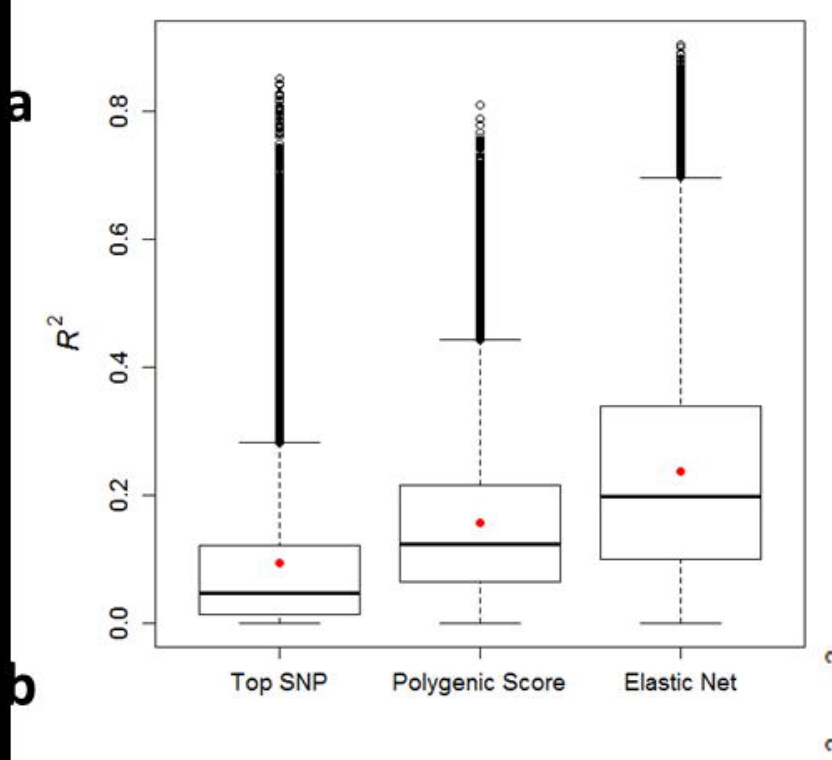

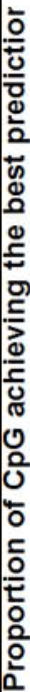

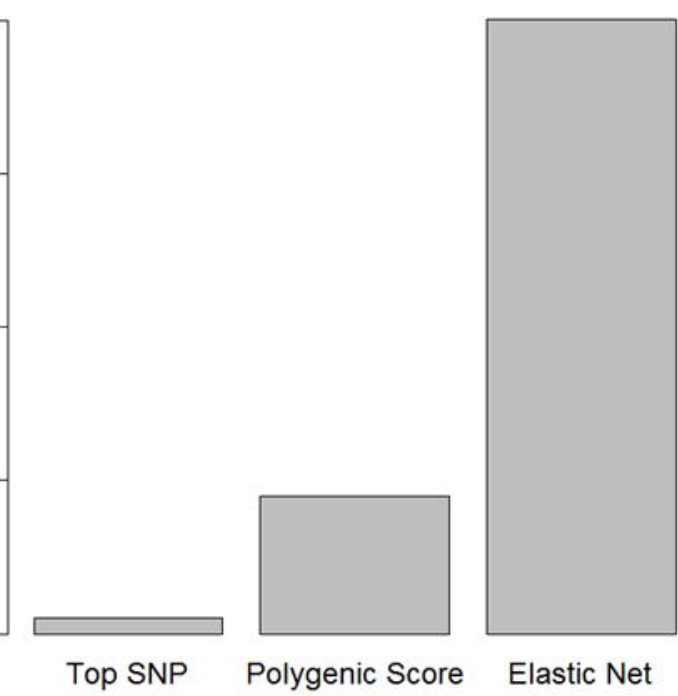

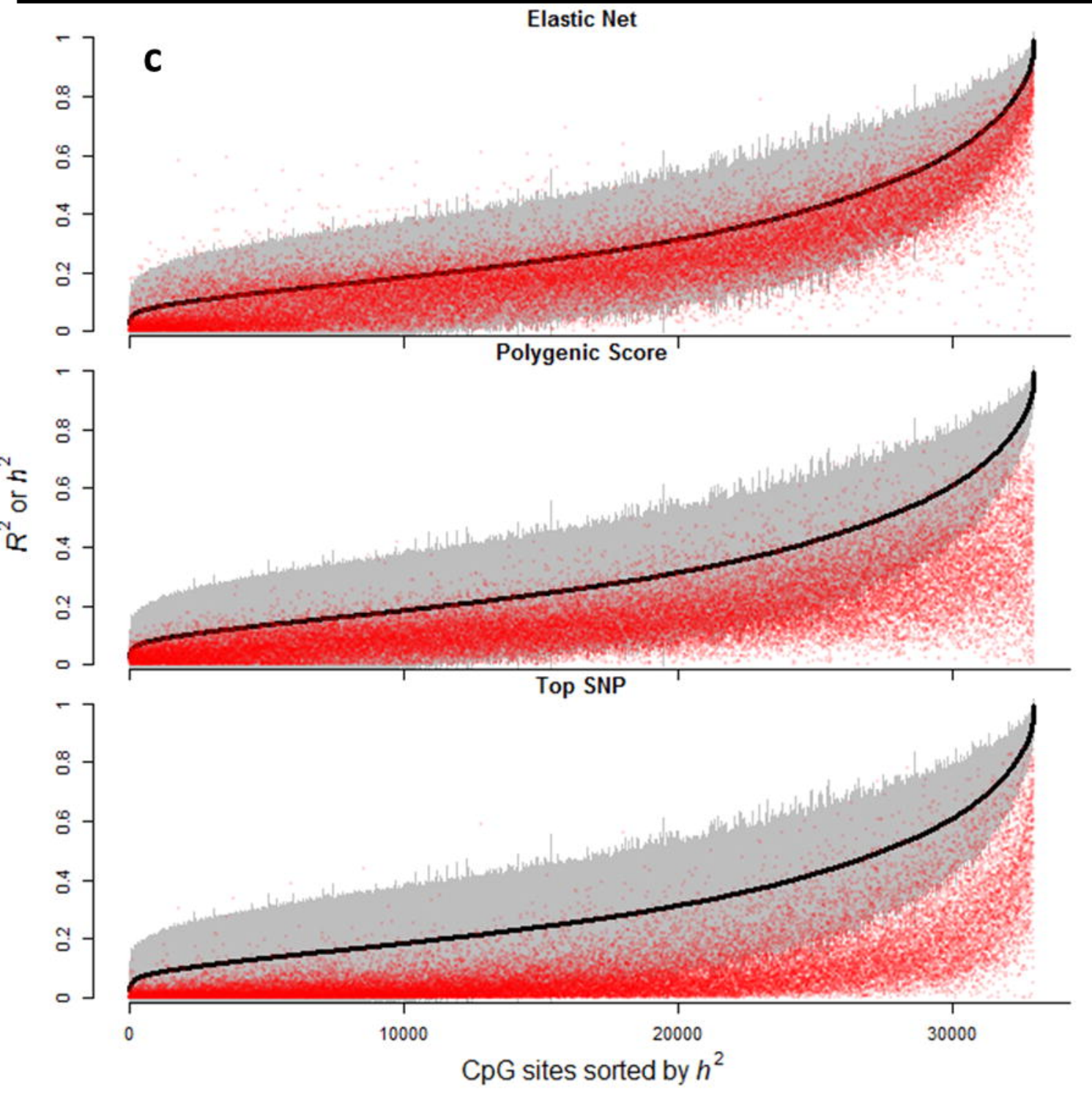



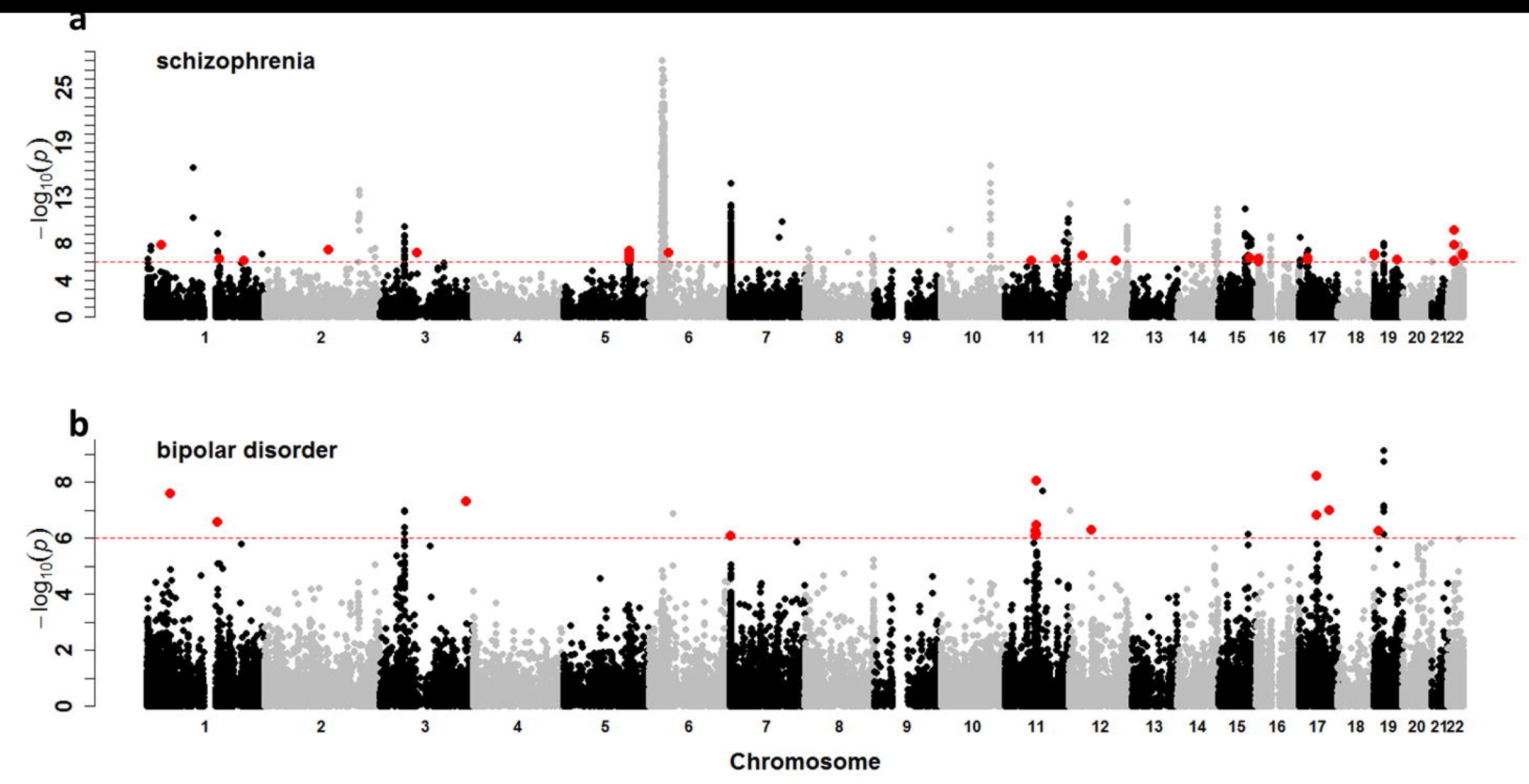
TBC1D10A SEC14L4

SEC14L2

SF3A1 ${ }^{2}$ SDC4P TCN2

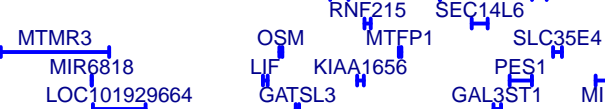

LOC101929664 GATSL3 H GAL3ST1 $\sqrt{\text { OSBP2 }}$ TUG1

HORMAD2

CDC157 DUSP18

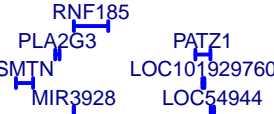

SELM LIMK2 EIF4EN|F1 PISD PRR14L

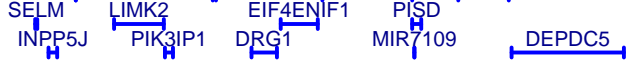
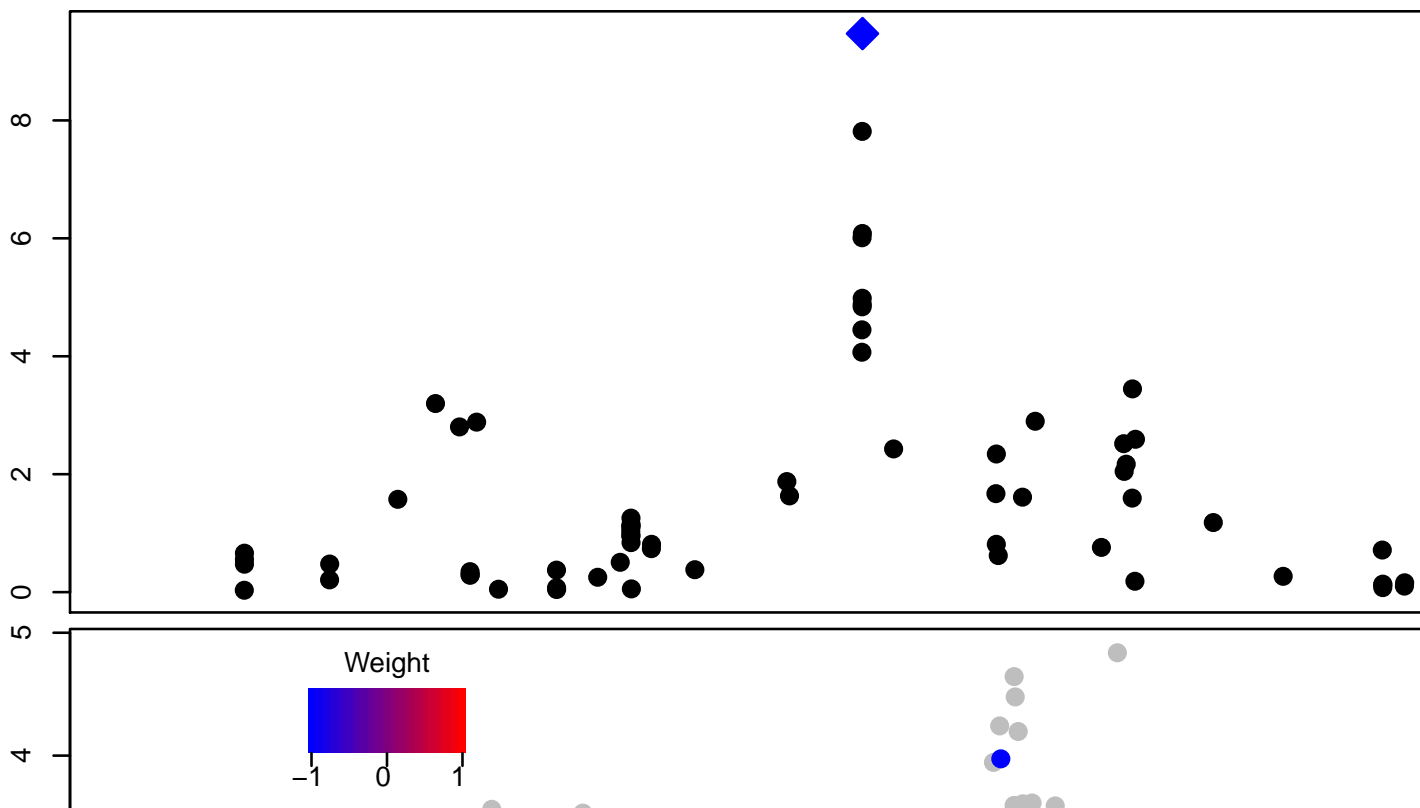

$\frac{2}{0}$
$\frac{0}{0}$

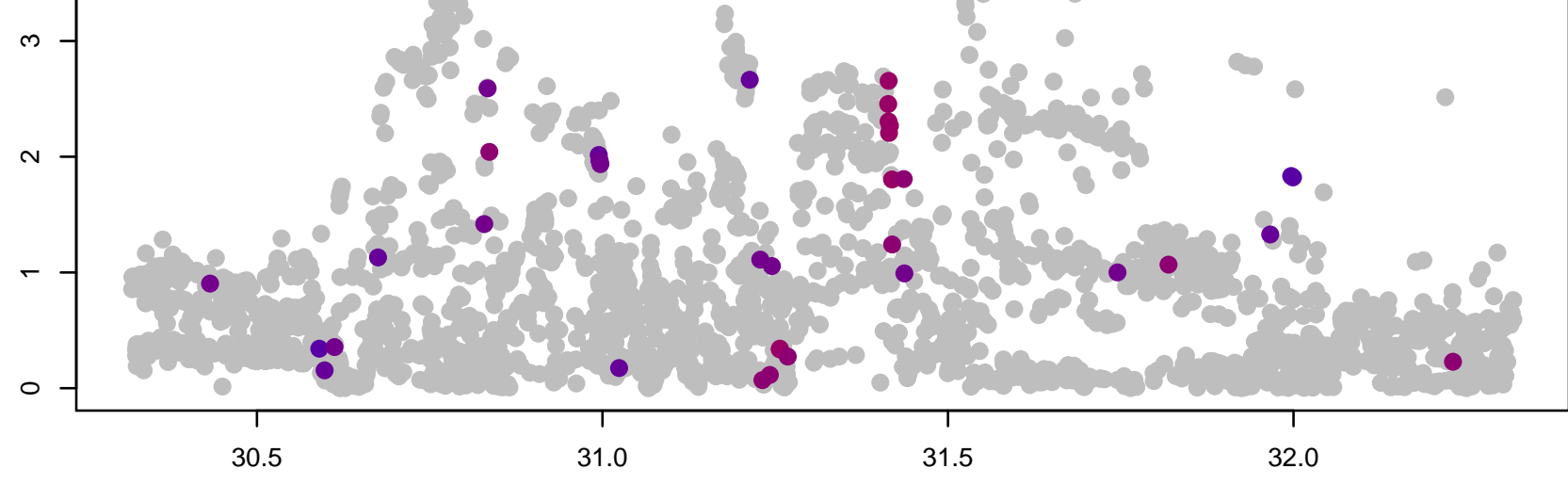

Chromosome position (Mb) 


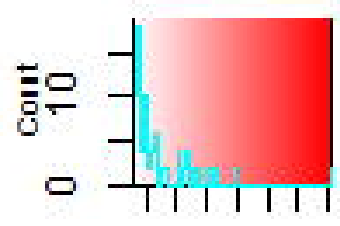

$5 \quad 20 \quad 35$

$-\log (\mathrm{p}$-value $)$

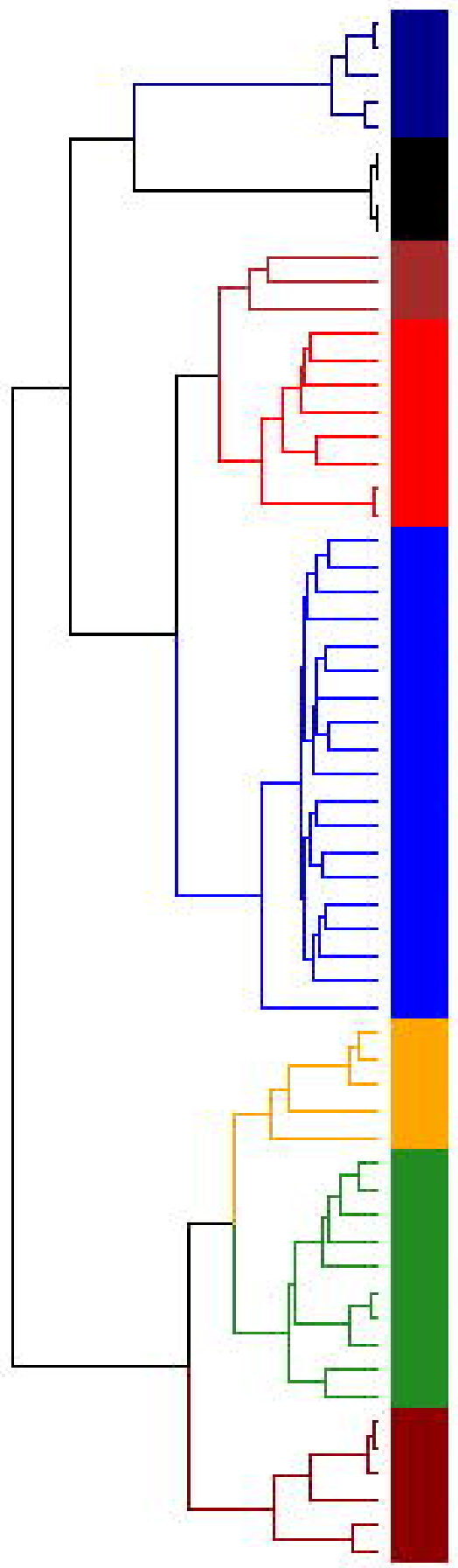

GO_MICROTUBULE_BUNDLE_FOFMATION

GO_AXONENE ASSEMBLY

GO'CEUL PFOE ECTION ORGANIZATION

GOMICFOTUBULE_BASED PFOCESS

GO-MICFOTUBULE-BASED-MOVENENT

GO-CELL CEUL ADFESION

GOBOLÖGICAL ADHESION

GO-CEUL CELL RDHESION VIA PLASMA NEMBRANE ADHESION_MOLECULES

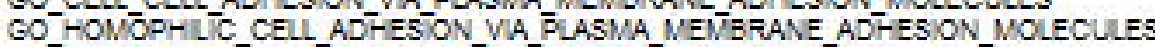

GO-POSITIVE REGULATION OF LFD BOSYNTHETIC PROCESS

GO ANTIGEN PROCESSNG AND PFESENTATION

PGE BP P 107

TGTTTAC MF30ASP MF30C_MF30D_MF306_MF30ESP

CTTGTAT-MF381

ACATTCC $M=1$ N $=205$

CACTGTG_M:123A_NF1260

ATATGCA MF448

GGGACCA MF133A M 71338

CHDB INSC HUNAN EFAN

CHD8-HNSC-HUMAN_MOUSE

GTTTGTT,MF 495

GGTGTGT MIF329

TGGTGCT_MIF29A_MIF296_MIF29C

GTGCCTT MI 505

TTTGCAC_MIR19A_MIR196

CAGCACT' MF512 $\overline{3}$ P

CACTTTG MIF520G MIF520H

ATGTAGC- MIF221 $\bar{M} / 2222$

ACCAATC-MIR509

AAGCACA MIF 218

CAGCTTT MIF320

AGGGCAG MIR 18 A

TCTCTCCDMIR185

TGCTTTG MIF330

TGAATGTDMIR181A_MIR181B_MIR181C_MIR181D

ATTCTTT MF186

GTATTAT-MIF3693P

TAATAAT MIR126

DENOVODB SCHZOPHFENA

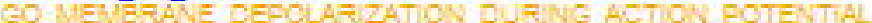

OW_CHANNELS_VOLTAGE_GATED_CALCIUN_CHANAEI

ION'CHANNELS ALL ION_CHANNELS

DENOVO DE ASTIS

$-G C, S C Z, P 10,4$

$F M F P$

CAV2_ION_CHANNELS_TRANSPORTERS

CONSTFANED

SYNAPTONE

$\mathrm{RBFOX2}$

$F B=O X 1$ FBFOX

POTENTALYY SYNAPTIC AUI

GENIC, INTOLERANCE, FV̈IS

PLO9

GO REGULATION_OF_SYNAPTIC PLASTICITY

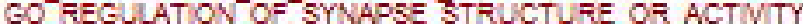

GO-MODULATION_OF_ SYNAPTIC_TRANSMSSION

GO-SYNAPSE_ORGANZATION

GO NEURONUSCULAF SYNAPTIC TRANSN SSION

GO_CEL_SURFACE_FECEPTOR_SGNALNG_PATHWAY_WVOVED_N_CEL_CEU_SIGNALNG 


\section{$R^{2}=0.85$}

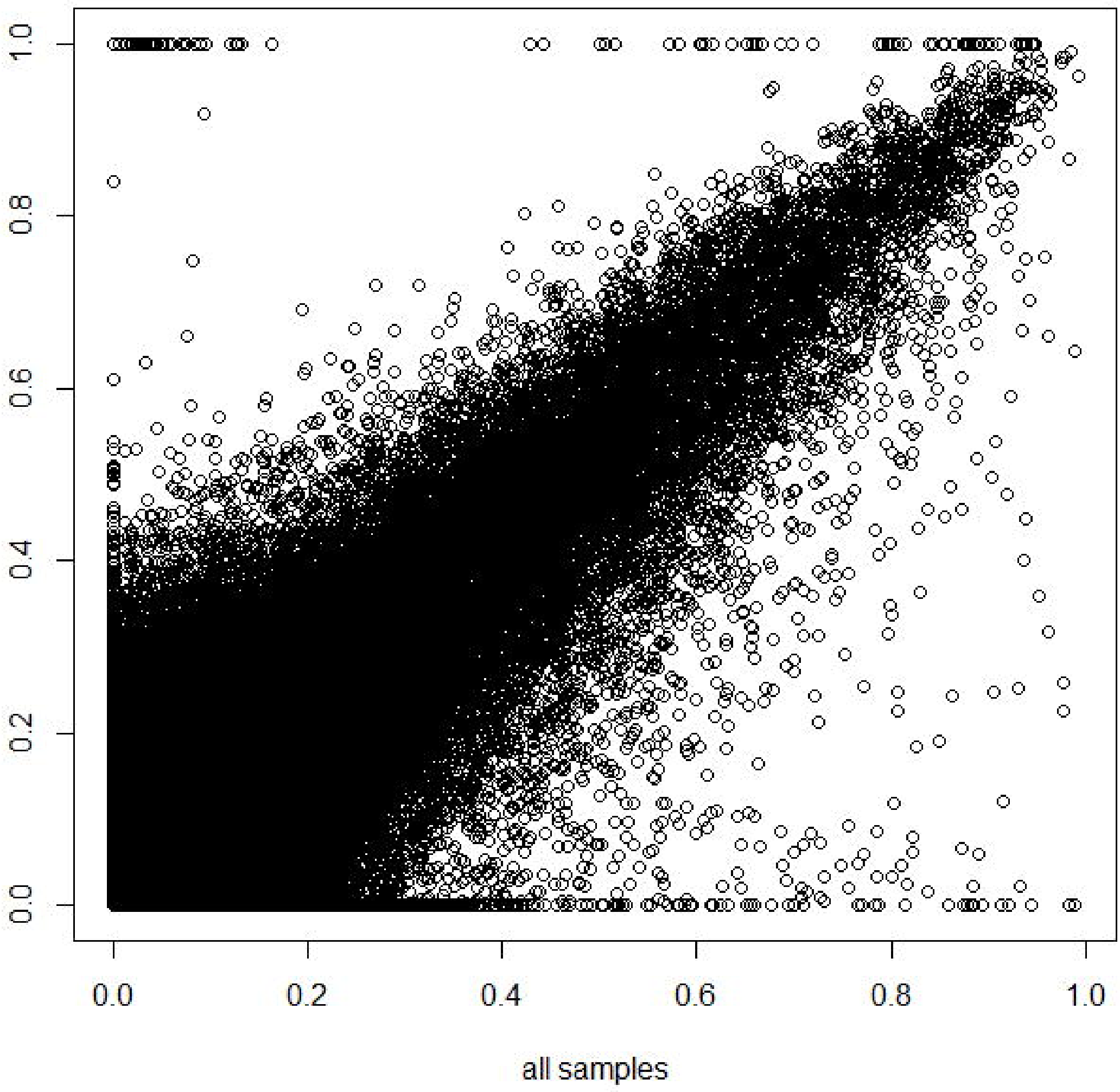


a

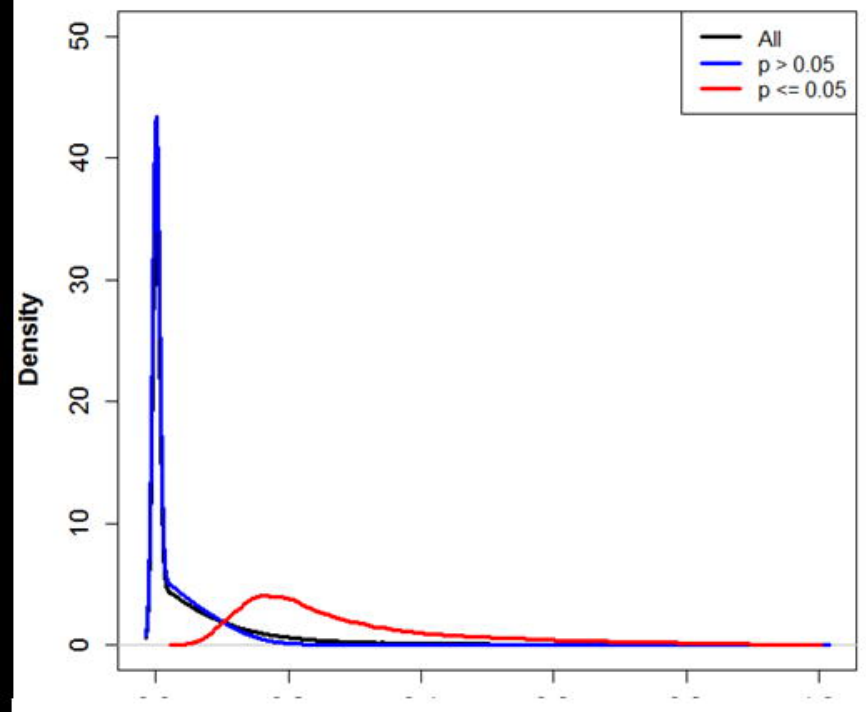

C

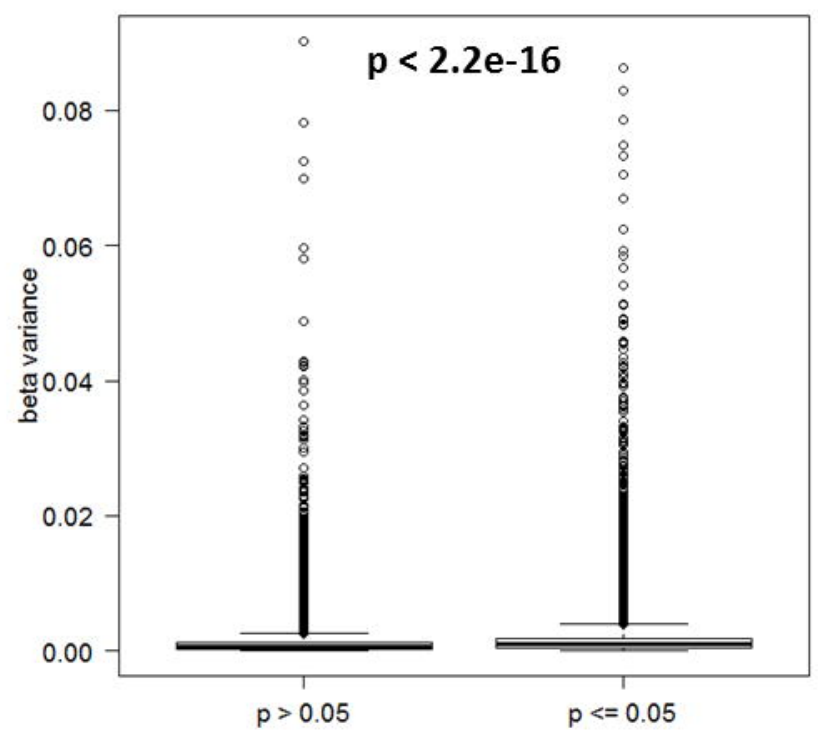

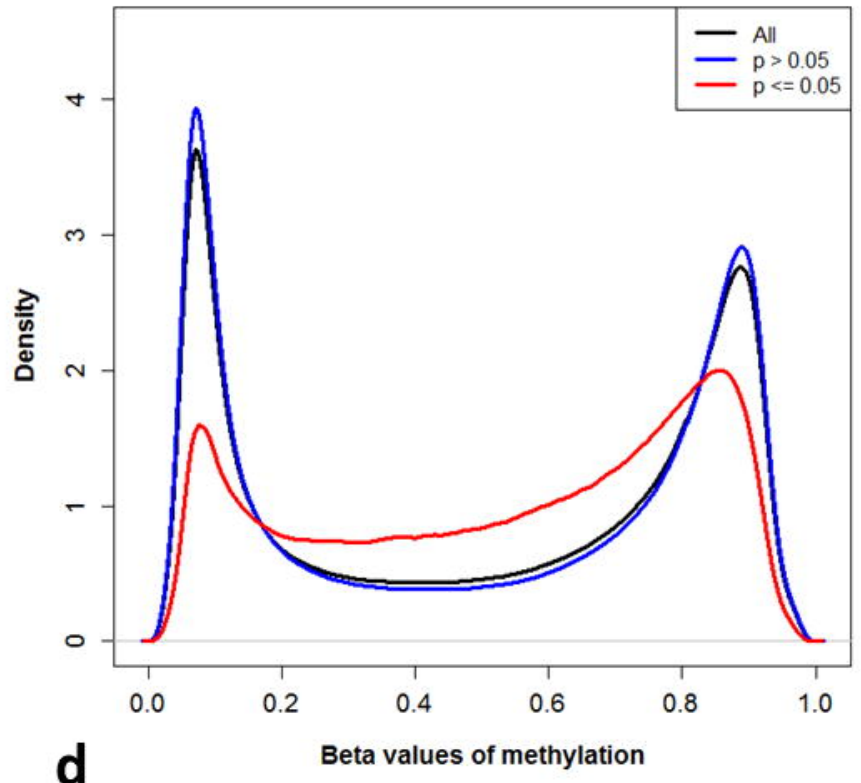

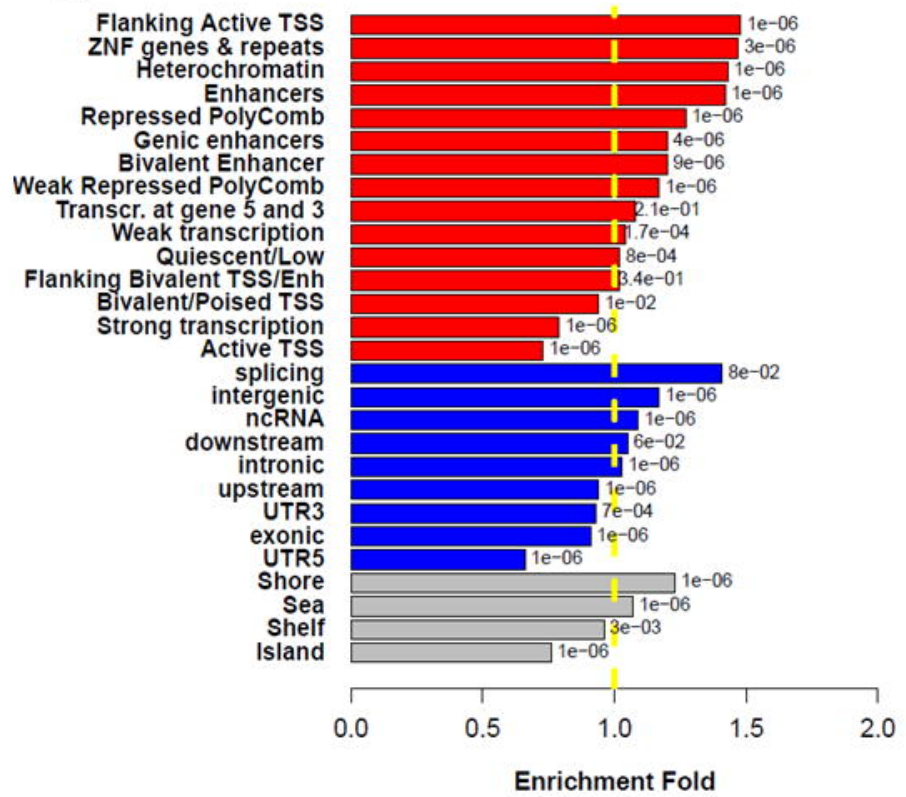


